\title{
Towards versatile legged robots through active impedance control
}

\section{Journal Article}

Author(s):

Semini, Claudio; Barasuol, Victor; Boaventura, Thiago; Frigerio, Marco; Focchi, Michele; Caldwell, Darwin G.; Buchli, Jonas

Publication date:

2015-06

Permanent link:

https://doi.org/10.3929/ethz-a-010604343

Rights / license:

In Copyright - Non-Commercial Use Permitted

Originally published in:

The International Journal of Robotics Research 34(7), https://doi.org/10.1177/0278364915578839

Funding acknowledgement:

601003 - Balance Augmentation in Locomotion, through Anticipative, Natural and Cooperative control of Exoskeletons. (EC) 


\title{
Towards versatile legged robots through active impedance control
}

\author{
Claudio Semini, Victor Barasuol, Thiago Boaventura, Marco Frigerio, Michele
}

Focchi, Darwin G. Caldwell, Jonas Buchli

\begin{abstract}
Robots with legs and arms have the potential to support humans in dangerous, dull or dirty tasks. A major motivation behind research on such robots is their potential versatility. However, these robots come at a high price in mechanical and control complexity. Hence, until they can demonstrate a clear advantage over their simpler counterparts, robots with arms and legs will not fulfill their true potential. In this paper, we discuss the opportunities for versatile robots that arise by actively controlling the mechanical impedance of joints and particularly legs. In contrast to passive elements like springs, active impedance is achieved by torque-controlled joints allowing real-time adjustment of stiffness and damping. Adjustable stiffness and damping in realtime is a fundamental building block towards versatility. Experiments with our $80 \mathrm{~kg}$ hydraulic quadruped robot HyQ demonstrate that active impedance alone (i.e. no springs in the structure) can successfully emulate passively compliant elements during highly-dynamic locomotion tasks (running, jumping and hopping); and, that no springs are needed to protect the actuation system. Here we present results of a flying trot, also referred to as running trot. To the authors' best knowledge this is the first time a flying trot has been successfully implemented on a robot without passive elements such as springs. A critical discussion on the pros and cons of active impedance concludes the paper. This article is an extension of our previous work (Semini et al. (2013)) presented at the International Symposium on Robotics Research (ISRR) 2013.
\end{abstract}

Key words: active impedance, legged locomotion, design and control, hydraulics, flying trot, quadruped robot

Claudio Semini, Victor Barasuol, Marco Frigerio, Michele Focchi and Darwin G. Caldwell

Dept. of Advanced Robotics, Istituto Italiano di Tecnologia (IIT), via Morego, 30, 16163 Genova, Italy e-mail: claudio.semini@iit.it, claudio@semini.ch, victor.barasuol@iit.it, marco.frigerio@iit.it, michele.focchi@iit.it and darwin.caldwell@iit.it

Thiago Boaventura and Jonas Buchli

Agile \& Dexterous Robotics Lab, ETH Zurich, Tannenstr. 3, 8092 Zürich, Switzerland e-mail: tboaventura@ethz.ch and buchlij@ethz.ch 


\section{Introduction}

Robots that combine legs and arms have the potential to become true everyday assistants to humans, and might eventually replace them for dangerous, dull or dirty tasks. While the legs will allow these robots to move with agility in any type of terrain accessible to humans and animals, their arms will allow them to execute tasks with human-like dexterity. Yet despite a long history and considerable efforts in research, legged robots are still mostly confined to research labs and a few prototype demonstrations. The motivation behind these efforts quite often cites their possible versatility; i.e. the use of one single robot in many different situations and especially in situations where other types of robots will not succeed (such as difficult terrains or human engineered environments). However, legged robots come at a high price in mechanical and control complexity. And until they can not demonstrate a clear advantage over their arguably simpler counterparts, their role will remain limited to research and they will not fulfill their promise of being versatile robotic assistants to humans. We can thus ask ourselves: What are major points that the robotics community needs to address to enable a breakthrough for versatile legged robots? In other words, what will allow us to design highly versatile and flexible robotic assistants that are truly useful to humans?

Today's most advanced robots with arms and legs are still very far from being very versatile and robust. In fact, the majority of today's legged robots struggle to move in even slightly rough terrain. This inability presents a stark contrast to human (or animal) capabilities and this discrepancy in performance has several reasons. Historically, robot arms - and later legs - were driven by stiff position-controlled joints. Interactions with the environment had to be carefully planned in the kinematic domain since information about the contact dynamics and forces could not easily be taken into account, and usually force and torque control was not available. While position control may be sufficient for most tasks undertaken by today's industrial robots, an autonomous machine will never be able to obtain a perfect map of the environment or a perfect robot state estimation. Thus, precise kinematic planning of contacts (e.g. footholds or grasp points) is not a feasible solution for robots that will have to move and interact in challenging and dynamically changing environments. Handling collisions and non-smooth interactions in a safe and robust manner has to be vital part of their list of specifications.

The physical laws governing interaction dynamics show that it is essential to control the joint torques and/or the contact forces during interactions with the environment (Hogan (1985a)), e.g. during locomotion on irregular terrain. Studies show that humans and animals are able to control joint torques thanks to antagonistically acting muscle pairs. The elasticity of the tendons in combination with muscle control allow to adjust both the passive and active joint impedance, respectively (Hogan (1984); Shadmer and Arbib (1992); Tee et al. (2009)). Active impedance for the hand or the foot is obtained by muscular control of co-contracting antagonistic muscle pairs (Franklin et al. (2003); Burdet et al. (2001); Selen et al. (2009)). According to Kandel et al. (2000) and Geyer and Herr (2010), this control naturally has a de- 
lay of few tens of milliseconds or more. During collisions, the passive compliance ${ }^{1}$ and damping in the tendons help to protect the actuation system during this delay. The smaller the delay, the less passive compliance/damping is needed to prevent damage.

In the recent decades, researchers have proposed several possible ways to cope with the interaction forces arising during contact with the environment. Some approaches use the passive dynamics of mechanical and pneumatic springs in the leg structure to govern the interaction dynamics (e.g. Buehler et al. (1998) and Raibert (1986)). The resonant frequency of the resulting spring-mass system can then be used to achieve a resonant hopping and running motion. Pratt and Williamson (1995) proposed the series elastic actuator (SEA) where (usually stiffer) springs are put in series with the actuator. The main functions of the spring in a SEA are to control the joint forces, absorb impact peaks and temporarily store energy. Springs are especially popular for electrically actuated robots, as they can also protect the gears $^{2}$ from getting damaged during collisions and non-smooth interactions.

These springs, however, introduce passive dynamics and low-frequency resonant modes into the system and therefore have to be tuned for a certain task. While this is fine for a single-purpose machine (e.g. a robot for highly efficient running), it drastically reduces the versatility and thus usefulness of a service robot for human environments. Even the normally stiffer springs of the SEA reduce the actuator bandwidth as a result of the resonant modes, and therefore make certain tasks, where a stiff and precise motion is required, difficult or impossible. This topic is further elaborated in Sect. 6.

To overcome this problem researchers have been working on variable stiffness actuators (VSA) that can vary the stiffness of each joint with the help of a (generally smaller) second actuator (see Vanderborght et al. (2013) for a recent review). While recent progress in this field has increased the range of adjustable stiffnesses (e.g. Tsagarakis et al. (2011)), the actuators are still bulky, complex and often cannot absorb high energy impacts due to the limited size of the springs.

Active impedance is a promising alternative that does not require any physical springs, because the required stiffness and damping is controlled by software and torque-controlled joints (e.g. impedance control by Hogan (1985b), operational space control by Khatib (1987), and virtual model control by Pratt et al. (2001)). Any stiffness and damping (within the limitations of the actuation and control system) can be selected in realtime either for the endeffector or for each joint independently (e.g. Boaventura et al. (2013)). This approach has most of the advantages of VSA without the above mentioned limitations. Boaventura et al. (2013) presented an experimental comparison study of active versus passive compliance and have shown that active impedance joints can emulate passive elements in the dynamic range needed for locomotion and interaction with the environment in general. The performance of the emulation is such that there is no relevant difference between the

\footnotetext{
${ }^{1}$ Compliance is the inverse of stiffness.

${ }^{2}$ Reduction gears are commonly required to amplify the low output torque of electric motors.
} 
dynamic behaviour of the actively controlled system and its fully passive 'template' system.

In this work we will demonstrate that active impedance can enable a legged robot to execute a wide range of different tasks in natural environments and thus increase its versatility and usefulness. In this context, we will present our previous work on our torque-controlled hydraulic quadruped robot HyQ (see Semini (2010) and Semini et al. (2011) for detailed descriptions of the robot). We will demonstrate the advantages and the potential of active impedance and torque-controlled robots within a series of new experiments: a flying trot on asphalt and an indoor treadmill, resonant hopping and squat jump landings. The flying trot and squat jumps will demonstrate the robustness and performance of the impedance controller in a very demanding situation due to the high frequency impacts at the moment of touch down. These test will also show how adjustable joint stiffness and damping can improve the locomotion performance. The resonant hopping trials will demonstrate the flexibility and versatility of the control concept.

The major contribution of this work is the presentation of a flying trot with an $80 \mathrm{~kg}$ quadruped robot with purely impedance-controlled legs and no springs in its mechanical structure. To the best knowledge of the authors no machine has achieved this before. In this paper we will use the success of this experiment as the basis for discussion of the importance of active impedance in legged robots for real-world tasks.

This paper first discusses the state of the art in the field of purely impedancecontrolled legged robots and machines that have successfully demonstrated a flying trot. Section 3 then introduces the active impedance controller used on our quadruped robot HyQ. The control required to implement a flying trot is explained in Sect. 4; and Sect. 5 presents the experimental results of flying trots, a resonant hopping motion with variable joint stiffness and squat jumps with variable joint damping. Finally, Sect. 6 discusses active versus passive impedance and how active impedance may help legged robots to achieve a technological and operational break-through. Section 7 concludes the paper with final remarks.

This article extends our previous work (Semini et al. (2013)) presented at the International Symposium on Robotics Research (ISRR) 2013 by (1) adding the results of two more experiments (a study of squat jumps and the impacts during the landing; and flying trot experiments on a treadmill with varying joint stiffnesses), (2) more examples of leg spring profiles and (3) a longer discussion including stiffnesses of various actuators.

\section{State of the Art}

We will discuss the state of the art of active impedance on legged robots and work related to experimental implementations of flying trots on quadruped robots. 


\subsection{Legged Robots with Active Impedance}

In this section we will focus on legged robots with active but no passive impedance, i.e. without any physical springs in their structure. For a more general and extensive review of impedance control in robotics, including fields such as haptics and manipulation, see Boaventura et al. (2012b, 2013). Within the literature there exist only a few examples of purely impedance-controlled legs with internal torque control loop. Ott et al. (2012) presented a bipedal walking robot with actuators based on the modular drives of the DLR-Lightweight-Robot-II (Hirzinger et al. (2001)). These actuator units are based on torque-controlled electric motors with integrated joint torque sensors. The robot successfully demonstrated walking on flat ground and stairs, as well as balancing and posture control. No highly-dynamic gaits like running have been demonstrated so far. Another electrically actuated robot with purely impedance- controlled legs is the MIT cheetah robot. Seok et al. (2012) presented a quadruped robot with joint torque control, implemented with electric motors with low gear ratio (5.8:1) and current control. No springs or torque sensing elements are needed in this approach (except an elastic spine for energy storage). The robot - supported by a boom - successfully demonstrated a running gait on a treadmill and showed reliable impedance control on joint level (Seok et al. (2013)). A similar approach was taken by Buchli et al. (2009) with LittleDog (developed by Boston Dynamics) that had joint level torque control based on electric motor current control. The paper showed how a feedforward torque term obtained by inverse dynamics can reduce the position gains and allow for successful disturbance rejection of unperceived obstacles. However, the high gear ratio, low control bandwidth and non-robust gears, made it very difficult to implement well controlled dynamic gaits. Another electric torque-controlled robot is the humanoid Roboray (developed by Samsung Electronics Co.). The pitch joints of the legs are composed of tendondriven stiff SEA actuators (Kim et al. (2012)). The robot has demonstrated walking and balancing but no more dynamic gaits like running or jumping.

There are also a few examples of hydraulically actuated robots with only active impedance. The US company SARCOS developed a few torque-controlled humanoid robots (e.g. CB Cheng et al. (2007)) that were sold to ATR in Japan, CMU in the USA and more recently to USC in the USA. The three research groups have shown balancing and simple stepping experiments on their robots (e.g. Hyon (2009); Stephens and Atkeson (2010); Herzog et al. (2014) at ATR, CMU and USC, respectively) but none have shown any more dynamic gaits like running for example. Hyon et al. (2013) at Ritsumeikan University have recently presented a lightweight hydraulic leg for research into agile legged locomotion. The leg demonstrated active impedance control on a vertical slider.

HyQ is a hydraulically-actuated quadruped robot developed at the Istituto Italiano di Tecnologia (Semini (2010); Semini et al. (2011)) with joint torque control based on torque sensors (Boaventura et al. (2012b)). This robot has successfully demonstrated various dynamic gaits ranging from fast walking $(2 \mathrm{~m} / \mathrm{s})$, jumping, rearing to trotting and balancing over rough and unstable terrain (Barasuol et al. (2013)). See Sect. 5.1 for a more detailed list of results and citations. Recent exper- 
imental studies by Boaventura et al. (2013) on a single leg of HyQ compared active versus passive impedance and showed that high-performance impedance controllers can emulate passive elements such as spring-dampers. Section 6.3 discusses some of these results. Section 5 of this paper is dedicated to show how active impedance can enable highly-dynamic and versatile locomotion.

Starting with the work on a series of novel fully torque controlled light-weight robots, at the German aeronautics and space research centre (DLR) (Hirzinger et al. (2001)), a lot of pioneering work has been done in investigating the potential of active torque controlled robots (e.g. demonstrating safety in De Luca et al. (2006)). While only recently (Ott et al. (2012)) legs have been built using this technology, already the early research on the LWR family of robots shares a lot of similarities with the herein presented approaches in terms of design of the actuation system as well as the low to mid-level control (Hirzinger et al. (2001)). The basic design principle is very similar: using a high performance actuator (a DC Brushless motor in the case of LWR robots) and a high precision load side torque sensor and position sensor. In the case of the LWR robots, the harmonic gears implement the, for torque controlled required, joint stiffness (cf. Focchi (2013) for a comparison of hydraulic and harmonic drive stiffnesses and Section 6 for a discussion relating this stiffness to SEA). Similar to our systems, on the actuator level a closed loop torque controller is employed that ensures high performance, stable torque tracking (Hirzinger et al. (2001); Albu-Schäffer and Hirzinger (2002)). Different approaches are studied and presented on the mid-level, amongst which Cartesian and joint-level impedance control schemes that are similar to our approach (Albu-Schäffer and Hirzinger (2002)). On a lower level, the biggest difference between the LWR systems and the herein used system to illustrate the potential of active impedance control are the choice of the actuation principle. In our case hydraulic cylinders and vane motors, in the LWR robots electric brushless DC motors are used. See Section 6 for discussion on the differences brought about by the different choices of actuator technologies. Furthermore, looking beyond the actuation and low-level control system, Ott et al. (2004) and Albu-Schäffer et al. (2007) proposed an elegant scheme for passivity based control of actively torque controlled robot, including both the actuator and the whole body control. These results can be in principle applied to other types of actuation systems (i.e. such as the one presented here) and is not a focus of the argument of this paper.

\subsection{Robots Running with a Flying Trot}

Next, we will discuss robots that have successfully demonstrated a flying (or running) trot. Note that we include robots with active and passive impedance, SEA, etc. in this overview. Raibert's quadruped robot from the CMU and later MIT leg lab was the first quadruped robot to demonstrate a flying trot (Raibert (1986)). Its prismatic legs had pneumatic springs in their structure that allowed the robot to run in resonance. Around 20 years later, Raibert et al. (2008) presented BigDog, 
a hydraulically-actuated quadruped robot. In one of the online videos (Boston Dynamics (2010) at 2:27), this robot demonstrated a flying trot. To date, no experimental results have been published. BigDog has torque-controlled joints and springs in the last segment of its legs. We believe that a combination of active and passive impedance is used in BigDog. StarlETH is a quadruped robot developed at the ETH Zurich with relatively stiff springs in series with its actuators (SEA) making it a fully torque-controlled robot (Hutter et al. (2012)). Gehring et al. (2013) recently presented results of StarlETH trotting with short flight phases. The Cheetah-cub is a $1.1 \mathrm{~kg}$ electric quadruped robot that recently demonstrated a flying trot (Sproewitz et al. (2013)). Its legs are designed around a spring loaded pantograph mechanism.

Note that all of the above-mentioned robots have passively compliant elements (mostly springs) in their legs.

A few other legged robots have shown running gaits, e.g. the biped MABLE using passive compliance with active force control (Hurst (2008); Sreenath et al. (2012)), KOLT with springs in the legs (Estremera and Waldron (2008)), MIT Cheetah (see Sect. 2.1 for more details), Boston Dynamics' Cheetah (no publication or information available). However, since some of their degrees of freedom are restricted by a boom or other guiding mechanism, they do not (yet) fully and convincingly demonstrate the mobility and versatility required for a useful service robot.

\section{Active Impedance}

With active impedance we mean that the mechanical impedance (1), is actively controlled and adjustable in software alone.

$$
f=K_{p}\left(x_{r e f}-x\right)+K_{d}\left(\dot{x}_{r e f}-\dot{x}\right)+K_{m}\left(\ddot{x}_{r e f}-\ddot{x}\right)
$$

where $f$ is the force; $K_{p}, K_{d}, K_{m}$ the stiffness, damping and inertia parameter, respectively; $x_{\text {ref }}$ a generalized position reference; and $x$ the actual position.

In our case we control the stiffness and damping only, as shown in (2), but did not implement inertia-shaping. While inertia-shaping may be crucial in impedance control for haptics, we do not consider it important for versatile legged robots, where appropriate inertia properties can be achieved by design.

$$
f=K_{p}\left(x_{r e f}-x\right)+K_{d}\left(\dot{x}_{r e f}-\dot{x}\right)
$$

To implement active impedance on HyQ, we use a cascaded control architecture as depicted in Fig. 1. In this control scheme, an outer impedance control uses the joint angular positions as feedback and produces a torque command as the output. Then, this torque command becomes the input reference for an inner torque control loop.

The high performance of the inner torque controller, obtained through low-level model-based techniques was essential to successfully achieve adjustable impedance through software, without the presence of real springs. For sake of completeness, we 
introduce in this section a few details of the model-based torque controller design already presented by Boaventura et al. (2012b,a, 2013) and Focchi et al. (2012).

Since the hydraulic pressure-flow dynamics is very non-linear, traditional linear controllers behave according to the design specifications only when close to the equilibrium point. To overcome this issue and to have the same response characteristics for all the actuator range of operation, we designed a nonlinear controller based on the input-output feedback linearization approach.

The controller output $u_{F L}$ (5), is composed of two main nonlinear terms: a velocity dependent term $f\left(x_{p}, \dot{x}_{p}\right)(3)$, which compensates for the natural load velocity feedback (Boaventura et al. (2012a)) in all the operating range (and not only around the operating point); and a pressure dependent term $g\left(P, x_{p}\right)(4)$ that compensates for the pressure-flow nonlinearities. These two terms are calculated based on the hydraulic pressure-flow dynamics as follows:

$$
\begin{gathered}
f\left(x_{p}, \dot{x}_{p}\right)=\beta A_{p}^{2}\left(1 / v_{a}+\alpha^{2} / v_{b}\right) \dot{x}_{p} \\
g\left(P, x_{p}\right)=\beta A_{p} K_{v}\left(\sqrt{\Delta P_{a}} / v_{a}+\alpha \sqrt{\Delta P_{b}} / v_{b}\right)
\end{gathered}
$$

where $x_{p}$ is the cylinder piston position; $\beta$ is the Bulk Modulus of the hydraulic oil; $A_{p}$ is the piston area; $v_{a}$ and $v_{b}$ are the volume of the cylinder chambers $a$ and $b$ respectively, including the pipe line volumes; $\alpha$ is the piston area ratio (e.g. $\alpha=1$ for symmetric cylinders); $K_{v}$ is the valve gain; and $\Delta P$ represents the pressure drop at each valve port.

$$
u_{F L}=\frac{1}{g\left(P, x_{p}\right)}\left(v-f\left(x_{p}, \dot{x}_{p}\right)\right)
$$

More details about the design of this controller for HyQ as well as some experimental results can be found in Boaventura (2013).

The inner torque controller permits a straightforward implementation of highlevel model-based control techniques, such as rigid body inverse dynamics, and gravity compensation. The output torques from these techniques can be easily added as a feed-forward torque to the torque reference command from the outer loop, as shown in Fig. 1. Some of these model-based techniques provide very convenient capabilities for performing robust locomotion in unstructured and partially unknown environments, as shown by Buchli et al. (2009). Essentially, such control methods allow the system's impedance to be lowered without degrading the position tracking performance.

The outer impedance loop defines the impedance characteristics of the robot, either set in joint or task space. The joint stiffness and damping can be implemented through a simple proportional derivative (PD) joint position controller. In this case, due to the presence of the inner torque loop, the proportional gain of the position control has units of $\mathrm{Nm} / \mathrm{rad}$, which corresponds to a rotational spring, and the derivative gain acquires the unit $\mathrm{Nms} / \mathrm{rad}$, which corresponds to a rotational damper. Therefore, by setting the joint proportional and derivative position gains it is possible to define the stiffness and damping of the robot. This joint-space 


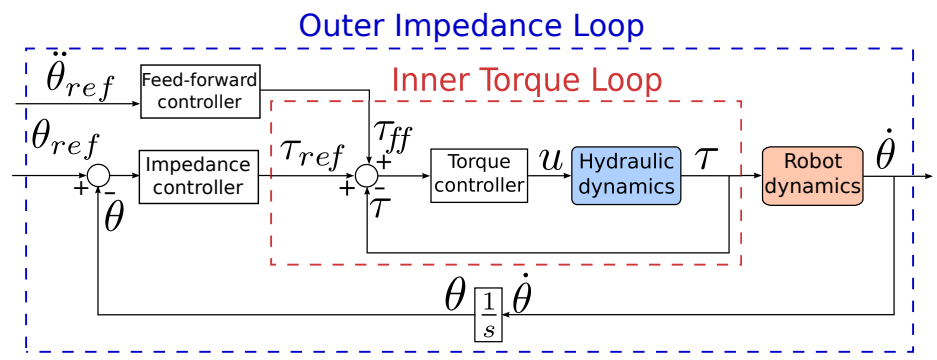

Fig. 1 Block diagram of the HyQ cascade impedance control architecture. It includes an outer impedance loop and an inner torque loop. The outer loop consists of a feedback controller and can also include a feed-forward controller such as rigid body inverse dynamics controller. The inner torque loop uses a feedback linearization for an increased tracking performance.

impedance scheme is used for the flying trot experiments described in Sect. 5.2 and 5.3, and the squat jump experiments of Sect. 5.5.

On the other hand, sometimes it might be more convenient to set the impedance at the end-effector instead of at the joints. A very intuitive way of defining a taskspace stiffness and damping is through the implementation of virtual components Pratt et al. (2001). As for the PD position controller mentioned above, these virtual components are also implemented in the impedance loop shown in Fig. 1. In HyQ, we designed a virtual spring-damper between the hip and the foot, see Fig. 2(a). The desired force $f$ created by these virtual components can be linear or nonlinear with respect to the stiffness, damping, and virtual prismatic leg length (Boaventura et al. (2012b)). Once the end-effector force $f$ is calculated, it is then mapped into joint-space through the Jacobian transpose of the kinematic transform from the virtual model coordinate system to the joint coordinate system. The use of the virtual prismatic leg is also a simple way of actively implementing the well-known spring loaded inverted pendulum (SLIP) model (Blickhan (1989)), which is a useful abstraction that describes the spring-like behaviour found in human and animal running and walking.

To demonstrate the ability of a HyQ leg to track a desired impedance profile, we implemented two different virtual elements: a linear spring and an exponential spring-damper, as depicted in Fig. 2(a). In this experiment, a single HyQ leg was fixed to a low-friction vertical slider which constrained the leg motion to the sagittal plane. The impedance tracking results are shown in Fig. 2(b). In this experiment, we submitted these two virtual elements to different dynamic conditions: the linear spring was tested by pushing the leg down by hand while it was standing on the floor; the exponential spring-damper, however, was investigated under a more dynamic situation where a $2 \mathrm{~Hz}$ sinusoidal excitation was applied to the spring natural length. As we see in Fig. 2(b), the impedance tracking is almost perfect under low-frequency perturbations and still acceptable in more dynamic circumstances. Section 6.3 shows a comparison between the HyQ virtual prismatic leg and a custom-made version of the same leg with a real spring-damper during a drop test. 


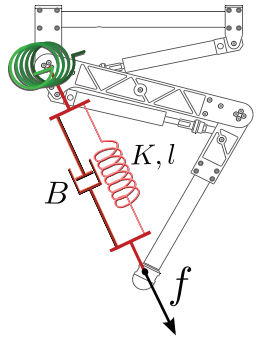

(a) Virtual elements

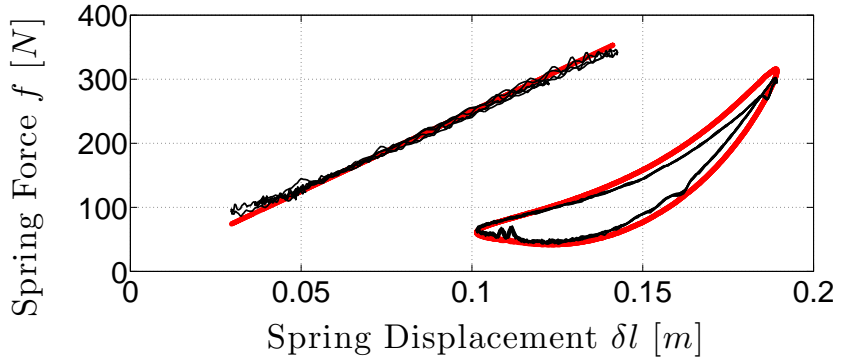

(b) Stiffness profile tracking

Fig. 2 HyQ virtual elements: (a) Drawing of a HyQ leg with virtual elements. A spring-damper connects the hip to the foot, creating a prismatic virtual leg. In the hip joint, a simple joint-space position PD control can be seen as a rotational spring-damper. (b) Spring force vs. displacement plot. Two different virtual elements were tested: a linear spring $(f=2500 \delta l)$ and an exponential spring-damper $\left(f=3 e^{25 \delta l}-50 \delta i\right)$. The red thick line represents the desired impedance profile, and the black thin line represents the actual impedance profile of the HyQ leg.

This task-space impedance controller is also used for the experiment presented in Sect. 5.4, where the stiffness of the linear spring is changed on the fly to create a resonant hopping with HyQ.

\section{Flying Trot Motion Generation and Control}

A trot is a gait in which diagonal leg pairs move simultaneously, alternating with the other pair of legs. A flying trot (or running trot) is a special case characterized by a ballistic body motion, i.e., by a period in which there are no legs in contact with the ground. The body flight phase depends on the time that a leg stays in contact with the ground (the stance phase) and the time that a leg takes to swing to the next foothold (the swing phase). The ratio between the stance period and the stride period (stance+swing) is called the Duty Factor, hereafter defined as $D_{f}$, and varies between 0 and 1. During trotting, if all the legs have a duty factor of less than 0.5 (i.e. swing phase longer than stance phase) then the body undergoes a flight phase for a certain time fraction of the gait cycle.

A comprehensive locomotion control framework is required to make a robot perform a stable flying trot. This control framework needs to integrate appropriate trajectory generation and body motion control in a closed loop fashion. Our recently presented Reactive Control Framework (RCF) (Barasuol et al. (2013)) implements these aspects and we adapted it to achieve a flying trot with HyQ. The RCF integrates the basic components for robot motion generation and robot motion control. No information about the environment, such as terrain surface level or obstacles, is required to achieve a basic robust (reactive) locomotion behaviour. 
Next, we will highlight some of the important features of the RCF in relation to the generation of a flying trot: the generated profile for the feet trajectories; the trajectory generator parameters; and how we choose such parameters to achieve a flying trot.

The generation of the reference trajectories for the feet is loosely inspired by the Central Pattern Generators (CPGs) of animals (Ijspeert (2008)), with the advantage of having intuitive parameters such as step length and step height instead of angular joint displacement for example. Ellipse-shaped trajectories (called primitives) are generated by a network of four non-linear oscillators, whose state represents the Cartesian coordinates of each foot (Barasuol et al. (2011)), as shown in Fig. 3 on the left. The oscillator parameters that define the aspect ratio of the ellipse are directly related to the step length $L_{s}$ and the step height $H_{s}$. Each oscillator has an angular frequency $w_{s}$, associated with the corresponding leg step frequency $f_{s} ; w_{s}$ might be different for the stance and swing phases, to achieve a duty factor different from 0.5 . Non-linear filters are coupled to the output of the network of oscillators to reshape the elliptical trajectories to semi-elliptical ones, to make the robot capable of adapting to the actual terrain profile. The non-linear filters reshape the primitive's trajectories according to an estimation of the foot position at touchdown; this information is either predefined, when the surface is well known, or computed from sensory information (for example using force sensors). The shape of the adapted trajectories are illustrated in Fig. 3 on the right.

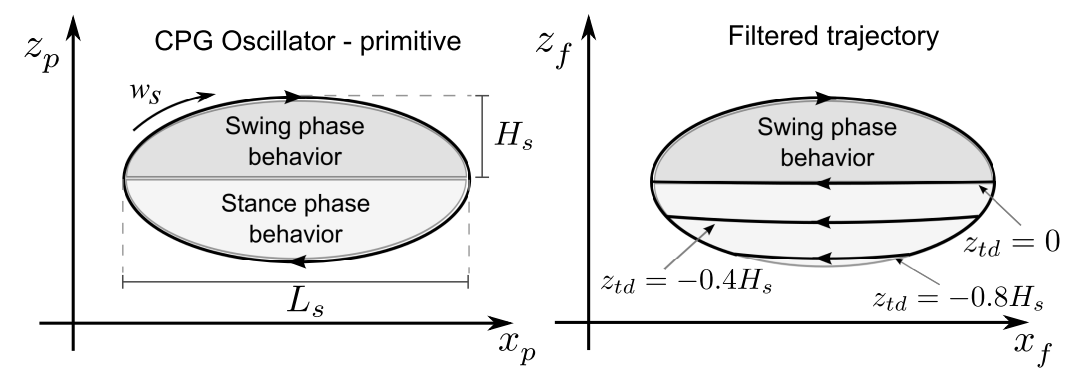

Fig. 3 The foot trajectory generated by the CPG oscillator (on the left) and the trajectory modulated by the non-linear filter (on the right). $z_{p}$ and $x_{p}$ are the reference coordinates of the primitive's trajectory, while $z_{f}$ and $x_{f}$ are the filtered references sent to the joint controllers (through inverse kinematics). $z_{t d}$ is the filter parameter which determines where the original elliptic trajectory has to be interrupted. (Figure modified from Barasuol et al. (2013))

The step depth parameter $z_{t d}$ affects the reshaping of the trajectory by determining at which height the ellipse has to be interrupted, as depicted in Fig. 3 on the right. The desired robot forward velocity $V_{f}$ determines the relative velocity of the foot with respect to the robot trunk, which is imposed during the flattened part of the semi-ellipse (i.e. during the stance phase). If a terrain map is available the swing-tostance transition can be planned in advance, reducing the impact forces. On the other hand, the feet trajectories can be dynamically adjusted even if the robot is walking blindly, e.g. by using feedback from the foot or joint force sensing, see Barasuol 
et al. (2013). This feature also makes the locomotion more robust with respect to poor state estimation.

In this paper we show experiments performed on flat ground. We consider the flat ground as a priori known surface and, therefore, we assume $z_{t d}=0$ for all the legs. With $z_{t d}=0$, the shape of the primitives becomes a half-ellipse.

During a flying trot the most important parameters are the step length $L_{s}$, the duty factor $D_{f}$, the desired forward velocity $V_{f}$ and the step frequency $f_{s}$. In the RCF approach all these parameters can be independently modulated. To achieve a stable spring-mass bouncing motion of the robot's centre of mass (COM), the robot's motion during the stance period needs to match the system's resonant frequency (defined by the robot's mass and leg stiffness). Selecting a proper duty factor and step frequency allows us to obtain a stance phase that matches the natural resonance period. With $D_{f}$ and $f_{s}$ defined, choosing a desired forward velocity $V_{f}$ consequently determines the value of the step length $L_{S}$ :

$$
L_{s}=\frac{V_{f} D_{f}}{f_{s}}
$$

In our flying trot, a key feature is the exploration of the independent parameter modulation capability of the RCF approach, which can be used to generate a variable swing velocity for the leg. The idea is to move the leg faster in regions where there is a low risk of impact with obstacles, while slowing it down near the expected touchdown regions, to reduce the impact forces. We obtain this leg behaviour by modulating the angular frequency of the primitives according to the collision-free region and the unknown touch-down region, without affecting the total swing period. See Fig. 4(a).

Figure 4(b) shows an example of Cartesian references for a flying trot run at 1 $\mathrm{m} / \mathrm{s}$ when the swing period in the collision-free region is chosen to be half of the swing period in the unknown touch-down region.

\section{Experimental Results}

We performed a series of experiments with our quadruped robot HyQ that uses only active (and no passive) impedance. After a description of the platform, we will present the results of four experiments: (1) a successful flying trot experiment on asphalt, (2) indoor flying trot trials on a treadmill, (3) resonant hopping and (4) a series of squat jumps. The results presented in this section illustrate the advantages and potentials of active impedance for legged robots. Videos of all experiments can be found in Extension 1. 


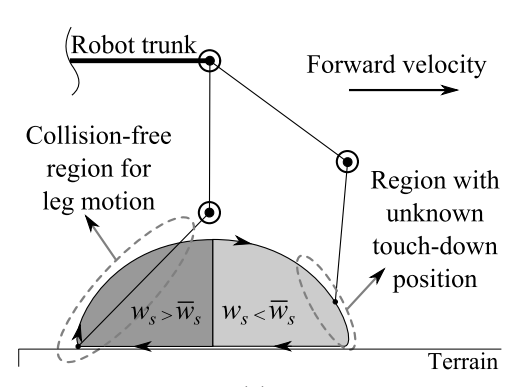

(a)

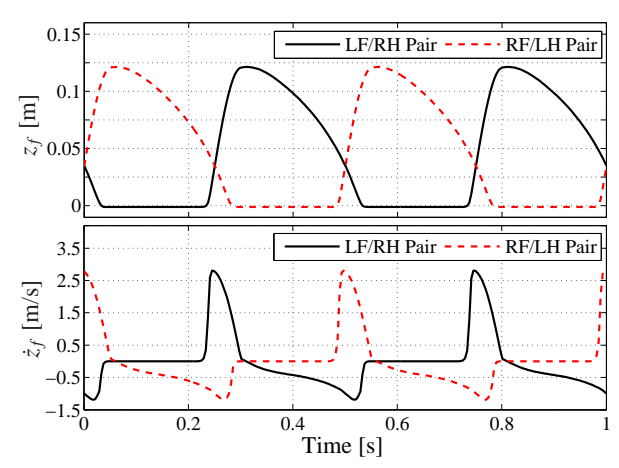

(b)

Fig. 4 Modulation of the angular frequency $w_{s}$. (a) In the collision-free region the angular frequency $w_{s}$ is greater than the average angular frequency, $\bar{w}_{s}$, of the swing phase. In the unknown touch-down region, $w_{s}$ is smaller than $\bar{w}_{s}$. (b) The plot shows the foot's relative position, $z_{f}$ (step height), and the corresponding velocity, $\dot{z}_{f}$, references for each pair of diagonal legs (LeftFront/Right-Hind and Right-Front/Left-Hind legs). The swing period in the collision-free region is chosen to be half of the swing period in the unknown touch-down region. The duty factor is 0.45 , the desired forward velocity is $1 \mathrm{~m} / \mathrm{s}$, the step frequency is $2 \mathrm{~Hz}$ and the step height is $0.12 \mathrm{~m}$.

\subsection{Experimental Platform $\mathrm{HyQ}$}

The platform used for these experiments is HyQ, Fig. 5a, a quadruped robot with hydraulically actuated joints (Semini (2010); Semini et al. (2011)). The machine weighs $80 \mathrm{~kg}$, is roughly 1 meter long and has a leg length of $0.78 \mathrm{~m}$ with fullyextended legs. All of its 12 degrees of freedom (DOF) are torque-controlled joints: The hip abduction/adduction (HAA) joints are driven by rotary hydraulic actuators with strain-gauge based torque sensors for torque control. All 8 joints in the sagittal plane (hip flexion/extension (HFE) and knee flexion/extension (KFE)) are actuated by hydraulic cylinders, Fig. 5b, that are connected to load cells for force measurement. High-performance servovalves (MOOG E024) enable joint-level torque control with excellent tracking (Boaventura et al. (2012b)) that led to the implementation of active impedance as described in Sect. 3. Note, that besides a thin $5 \mathrm{~mm}$ layer of stiff rubber at the feet, there are no passive stiffness/damping elements (e.g. springs) present anywhere in the robot's leg structure. See Sect. 6.4 for a discussion on compliance inside and outside the robot structure. Table 1 lists the main specifications and features of the robot.

Since 2011 HyQ has demonstrated a wide repertoire of static and dynamic motions ranging from squat jumps (Semini et al. (2012)), rearing (Boaventura et al. (2012b)), step reflexes (Focchi et al. (2013)), walking trot over flat, inclined and rough terrain (indoors and outdoors), balancing under disturbances (Barasuol et al. (2013)), perception-enhanced trotting and crawling (Havoutis et al. (2013); Bazeille 


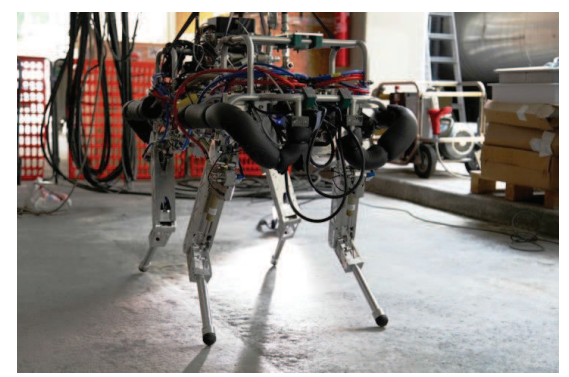

(a) Picture of HyQ

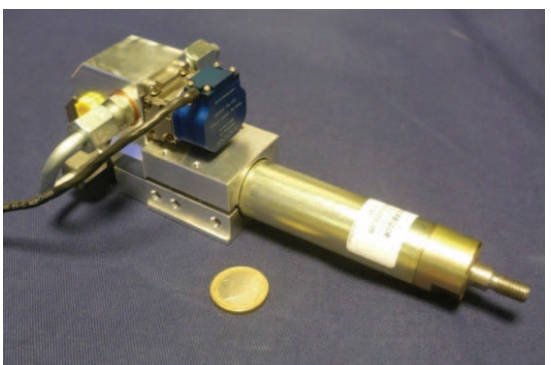

(b) Picture of HyQ's hydraulic actuator unit

Fig. 5 (a) Picture of IIT's quadruped robot HyQ. (Picture credit: Agnese Abrusci, IIT); (b) Picture of HyQ's hydraulic actuator unit consisting of a double-acting cylinder, manifold and servovalve.

Table 1 Overview of the specifications and features of the HyQ robot.

\begin{tabular}{ll}
\hline Properties/Features & Values \\
\hline dimensions & 1.0m x 0.5m x 0.98m (LxWxH) \\
link lengths & HAA-HFE: $0.08 \mathrm{~m}$ \\
& HFE-KFE: $0.35 \mathrm{~m}$ \\
& KFE-foot: $0.35 \mathrm{~m}$ \\
weight & 80kg \\
active DOF & 12 \\
hydraulic actuation (HAA) & double-vane rotary actuators \\
hydraulic actuation (HFE, KFE) & double-acting asymmetric cylinders \\
& (Hoerbiger LB6 1610 0080) \\
motion range & $90^{\circ}$ (HAA), 120 (HFE, KFE) \\
maximum joint torque (HAA) & $120 \mathrm{Nm}$ (peak torque at 20MPa) \\
maximum joint torque (HFE, KFE) & 181 Nm (peak torque at 20MPa) \\
position sensors & relative encoder, 80000cpr in all joints \\
& (AVAGO AEDA3300 BE1) \\
torque sensors & custom torque (HAA), 5kN loadcell (HFE, KFE) \\
& (Burster $8417-5000)$ \\
perception sensors & IMU, stereo camera, lidar \\
onboard computer & PC104 stack with Pentium CPU board \\
joint control (rate) & position and torque (1kHz) \\
locomotion skills & walking (crawl, trot), running (flying trot, presented in \\
& this paper), hopping, squat jumping, rearing \\
\hline
\end{tabular}

et al. (2014)), to an optimized crawl gait for walking on stairs and stepping stones (Winkler et al. (2014)). A summary video of these results is available online (IIT, ADVR, DLS lab (March 2014)). 


\subsection{Flying Trot on Asphalt}

We conducted several experiments of a flying trot with HyQ on asphalt using the approach presented in Sect. 4.

Figure 6 shows the HFE and KFE joint torque plots of the four legs and the vertical ground reaction forces. The duty factor during this experiment was set to 0.45 , the step height $0.12 \mathrm{~m}$, forward velocity $1.3 \mathrm{~m} / \mathrm{s}$, step length $0.28 \mathrm{~m}$ and the joint-level active stiffness $300 \mathrm{Nm} / \mathrm{rad}$ for the hip and knee flexion/extension joints.

Note that the joint torques of all four legs stay inside the maximum torque limits ${ }^{3}$ of $181 \mathrm{Nm}$ demonstrating that active compliance can successfully absorb the high impacts during running and cope with these collisions. The plots also show that the KFE joint torques and ground reaction forces go to zero between the stance phases of the diagonal leg pairs. This illustrates that the robot was indeed in flight phases. To the best of our knowledge no other robot has successfully shown a robust flying trot with active impedance only, i.e. without passive elements such as springs in its legs.

\subsection{Flying Trot on Indoor Treadmill}

For the flying trot experiment presented above we first selected the kinematic references (e.g. forward velocity, step height, step frequency) based on previously obtained parameters that are suitable for a walking trot (duty factor $>0.5$ ) as described in Barasuol et al. (2013). Subsequently, the duty factor was gradually reduced from 0.55 to below 0.5 to induce a flying trot. Finally, to create a robust flying trot, we matched the (virtual) spring-mass system's resonance to the gait frequency by handtuning the joint stiffness of HFE and KFE.

To study the influence of the joint stiffness on the flying trot robustness, we performed a series of experiments on a custom-made indoor treadmill. The joint damping $K_{d}$ of all joints is set to $6 \mathrm{Nm} \cdot \mathrm{s} / \mathrm{rad}$. While the stiffness of HAA was kept constant at $K_{p}=300 \mathrm{Nm} / \mathrm{rad}$, the HFE and KFE stiffness is adjustable. Fig. 7 shows the vertical ground reaction force plots for $K_{p}=350,250$, and $150 \mathrm{Nm} / \mathrm{rad}$.

The plots show that high $K_{p}$ values lead to higher force impact peaks at touchdown, as can be seen in the beginning of the stance phases of the first subplot. With decreasing $K_{p}$ the robot cannot maintain a robust flying trot. This is illustrated by disappearing flight phases in the lowest subplot. The robot shows an increasingly asymmetric, limping behaviour (see also Extension 1). The figure shows that stiffness values for HFE and KFE in the range of 250 to $350 \mathrm{Nm} / \mathrm{rad}$ lead to a robust flying trot for the $80 \mathrm{~kg}$ HyQ robot. Suitable stiffness and damping values depend on the robot weight, kinematics and the task. We discuss the selection of optimal impedance values at the end of Sect. 5.5.

\footnotetext{
${ }^{3}$ Note that we recently increased the hydraulic system pressure of the HyQ robot to $20 \mathrm{MPa}$, increasing the maximum torque of the hip and knee flexion/extension joints to $181 \mathrm{Nm}$.
} 


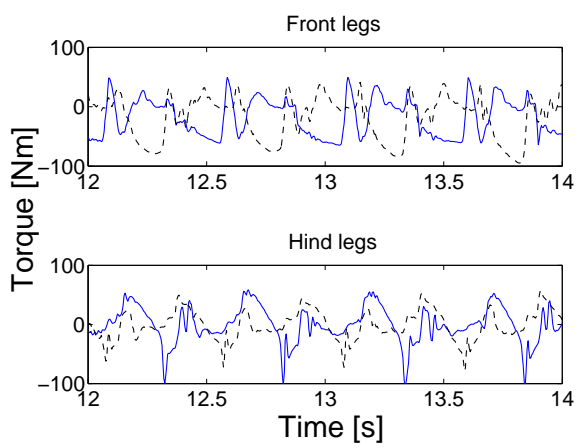

(a) Torque at the hip joints

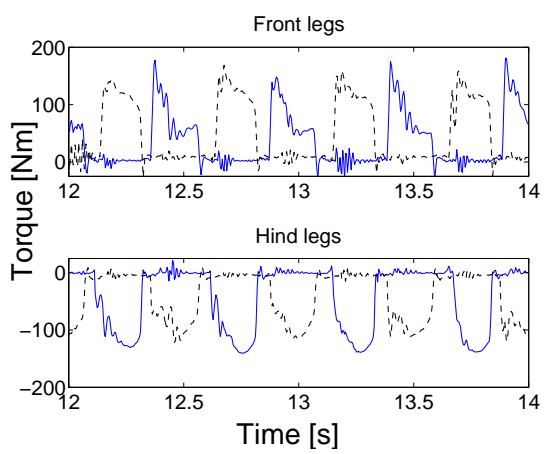

(b) Torque at the knee joints

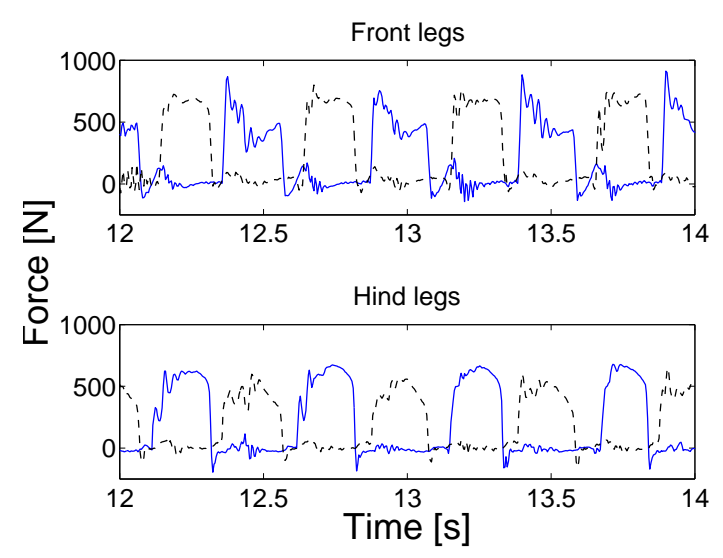

(c) Vertical ground reaction forces

Fig. 6 Force profiles during the flying trot experiment; the blue solid lines refer to the left legs, the black dashed lines to the right legs. (a) This plot shows the torques at the four hip joints (HFE) of the robot; (b) This plot shows the torques at the four knee joints (KFE) of the robot; the short intervals during which all the torques are close to zero are due to the flight phase. (c) This plot illustrates the ground reaction forces during the same time interval, estimated from the torques at the knees and hips with the transpose of the Jacobian.

\subsection{Resonant Hopping Experiment}

In this section we show HyQ's ability to change the virtual spring stiffness on the fly, to achieve a resonant hopping motion. To achieve this, we implemented a virtual linear spring-damper for all four legs of HyQ as shown in Fig. 2(a). The length of the virtual linear springs $(l=0.58 \mathrm{~m})$ is varied sinusoidally (peak to peak: $0.05 \mathrm{~m}$ ) at a constant frequency of $1.6 \mathrm{~Hz}$. During the experiment, the stiffness of the virtual springs is linearly changed from $K=2000 \mathrm{~N} / \mathrm{m}$ to $K=5000 \mathrm{~N} / \mathrm{m}$.

As shown in Fig. 8, after $1 \mathrm{~s}$ the spring stiffness starts to increase and, consequently, the amplitude of the ground reaction force oscillations grows due to reso- 


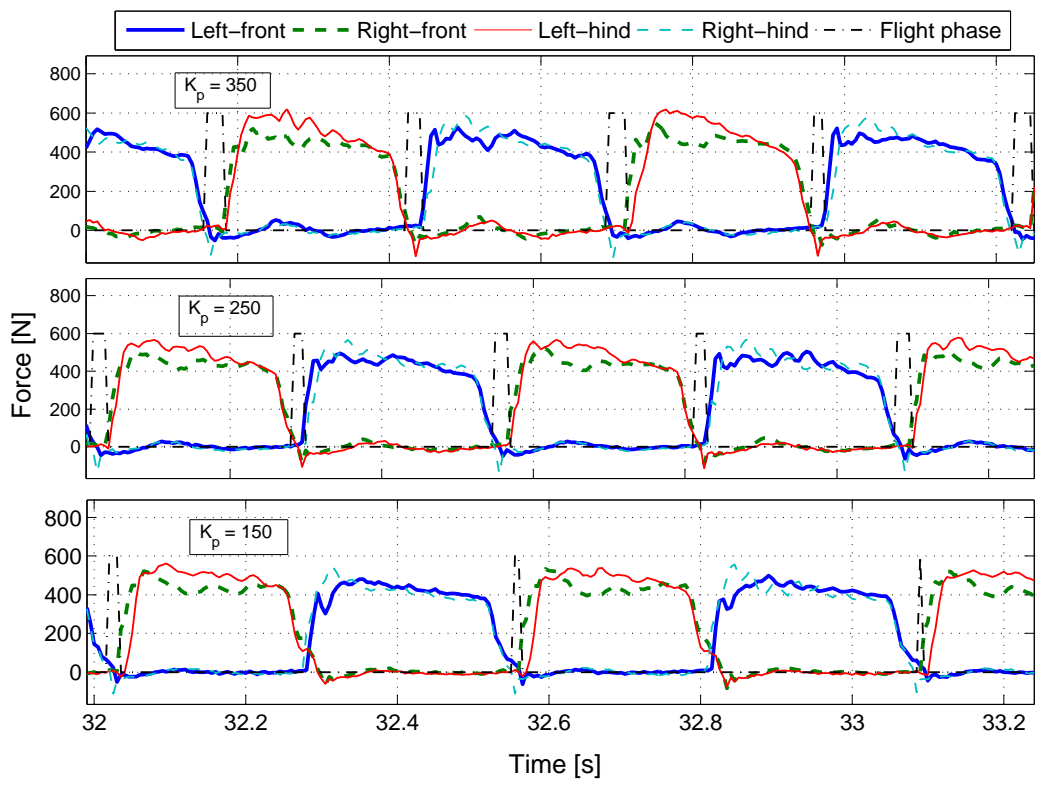

Fig. 7 Force plots of flying trot experiments on an indoor treadmill. The plots show the vertical ground reaction forces of the four legs for three trials with different stiffness values for the HFE and KFE. Top: $K_{p}=350 \mathrm{Nm} / \mathrm{rad}$, middle: $K_{p}=250 \mathrm{Nm} / \mathrm{rad}$, bottom: $K_{p}=150 \mathrm{Nm} / \mathrm{rad}$. Note that the stiffness is decreasing from top to bottom. The forces are estimated from the torques at the knees and hips with the transpose of the Jacobian. The black dash-dotted line indicates the presence of a flight phase if $\neq 0$.

nant effects. We show the ground reaction force for the left front (LF) leg in the top plot. When the stiffness increases to the point that the spring-mass system resonates with the frequency of the sinusoidal spring length excitation, the robot starts to hop and the ground reaction forces go to zero during the flight phase (all four legs in the air). The resonance peak occurs at about $10 \mathrm{~s}$, when the stiffness is around 3800 $\mathrm{N} / \mathrm{m}$.

\subsection{Squat Jumps with Adjustable Damping}

Leg compliance and damping are important for versatile legged robots, especially if they are designed to perform highly dynamic motions such as jumping and running. In this section we show an example of how active impedance can be used as a "degree-of-freedom" to manage the robot-environment interaction forces while maintaining a position objective such as body posture and/or COM position. HyQ performed a number of squat jumps to create repeatable impacts on the robot's legs during the landing. To reduce the forces transmitted to the robot structure, we stud- 


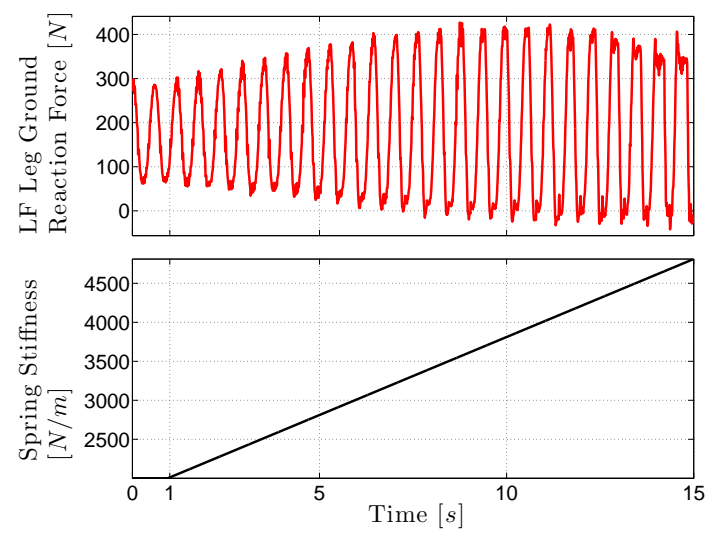

Fig. 8 Experimental results of resonant hopping: We implemented a hopping motion by exciting the HyQ robot in resonance by varying the virtual legs stiffness. The top plot shows the ground reaction force for the left front (LF) leg, which reaches zero after around $9 \mathrm{~s}$ demonstrating the presence of a flight phase. The bottom plot presents the linear change in stiffness applied to the legs.

ied the influence of joint stiffness $K_{p}$ and damping $K_{d}$ on the impact force and settling time. Additionally, we present the results of a simple control law that adjusts the joint damping during the landing.

In all experiments the robot starts in a symmetric default position with all four legs on the ground. A vertical force impulse is then applied to the robot's COM (4000N peak and 300ms duration). The robot controller maps this force reference from COM space to joint space, creating a feedforward torque term for the joint torque controller. After lift-off the robot enters a parabolic flight phase that lasts around $350 \mathrm{~ms}$ for the given force impulse. The results of over 100 repetitions with the same jump force profile demonstrated that the experiment is repeatable, by providing equal kinetic energy before the landing, and is thus suitable for this study. This allowed us to compare the measured knee joint torques obtained during the trials with different joint stiffness and damping values.

Figure 9 shows the results of a selection of 5 experiments where knee torques and $\mathrm{COM}$ vertical position are evaluated. The COM position is estimated through the leg kinematics. The first 4 experiments have the following joint stiffness/damping pairs $K_{p}, K_{d}$ for HFE and KFE: $300 \mathrm{Nm} / \mathrm{rad}, 6 \mathrm{Nm} \cdot \mathrm{s} / \mathrm{rad}$ (black thin solid line), 150 $\mathrm{Nm} / \mathrm{rad}, 6 \mathrm{Nm} \cdot \mathrm{s} / \mathrm{rad}$ (blue dashed), $150 \mathrm{Nm} / \mathrm{rad}, 12 \mathrm{Nm} \cdot \mathrm{s} / \mathrm{rad}$ (red dash-dotted), 200 $\mathrm{Nm} / \mathrm{rad}, 12 \mathrm{Nm} \cdot \mathrm{s} / \mathrm{rad}$ (green thick solid).

From the results it can be seen that a higher joint stiffness $K_{p}$ reduces the steadystate error of the COM vertical position (created by gravity forces), but leads to a higher joint load when the leg changes from compression to extension (and the body reaches the lowest point). It can even create rebounding, as shown with the black thin solid line of the top plot. Higher $K_{d}$ values dampen the system response (by stabilizing the system as quickly as possible), but lead to a higher joint load at the moment of touch-down when the velocity error state reaches its maximum value. 


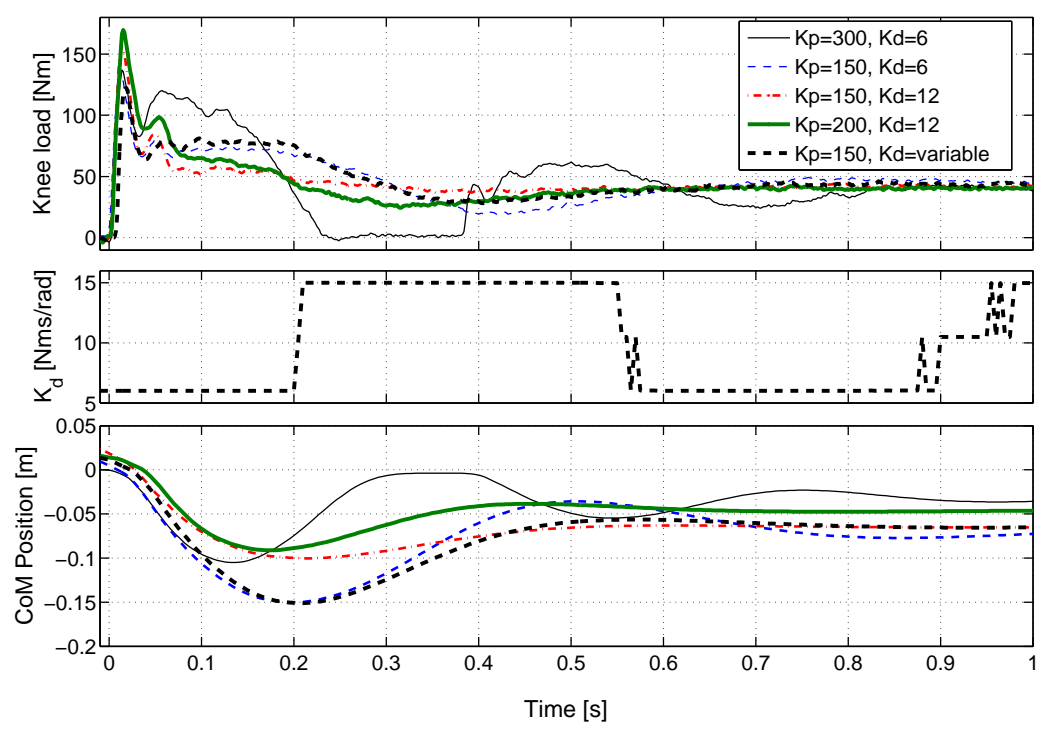

Fig. 9 The plot shows the results of 5 squat jump trials performed with different stiffness and damping pairs $K_{p}, K_{d}$, where each pair is equally set for the HFE and KFE joints: 300, 6 (black thin solid line), 150, 6 (blue dashed), 150, 12 (red dash-dotted), 200, 12 (green thick solid), 150, variable (black thick dashed). The black thick dashed line is associated to the trial with variable damping for the HFE and KFE joints. The middle plot shows the value of the modulated joint damping $K_{d}$ while the bottom plot shows the estimated COM vertical position during the landing, where COM $=0$ is the desired vertical position.

The red dash-dotted and green thick solid lines of the top plot of Fig. 9 show that even with different $K_{p}$ gains $\left(K_{p}=150 \mathrm{Nm} / \mathrm{rad}\right.$ and $\left.K_{p}=200 \mathrm{Nm} / \mathrm{rad}\right)$ the initial impacts are stronger than in the trials with $K_{d}=6 \mathrm{Nm} \cdot \mathrm{s} / \mathrm{rad}$.

A modulation of the damping value can therefore combine the advantage of low damping to reduce the initial impact peaks, with the higher damping to dampen the system response after the impact. The black dashed line shows the result of a landing with fixed $K_{p}=150 \mathrm{Nm} / \mathrm{rad}$ and variable damping, that is modulated according to the knee joint velocity as follows:

$$
K_{d}=K_{d 0}+\Delta K_{d} \sigma(k n e e \text { joint velocity })
$$

where $K_{d 0}=6 \mathrm{Nm} \cdot \mathrm{s} / \mathrm{rad}, \Delta K_{d}=9 \mathrm{Nm} \cdot \mathrm{s} / \mathrm{rad}$ and $\sigma(\mathrm{arg})$ is a sigmoid function that continuously ranges from 0 to 1 according to its argument $\arg$. The following sigmoid function is used for the active impedance modulation:

$$
\sigma(\arg )=\frac{1}{1+e^{-K_{s} \arg }}
$$

where the sigmoid constant $K_{s}$ is equal to 50. This leads to a continuous modulation of the damping from 6 to 15 depending on whether the leg is in a compression or extension phase after the impact. The black dashed line of the top plot in Fig. 
9 shows how a low initial impact peak can be combined with a low settling time. While this is a very simple control law, more sophisticated modulations are possible, such as for example the skyhook controller that we implemented in Kostamo et al. (2013) for a semi-active magneto-rheological damper.

The stiffness and damping values used for the experiments presented above are obtained experimentally. They work well for a robot of the mass and kinematics of HyQ (see Table 1). The relation between leg stiffness and animal running performance is studied by biologists. Lee et al. (2014), for example, analyzed the leg stiffness of mammals during bouncing gaits and provides a metric to estimate their leg stiffness in relation to body mass. A scaling study resulting in dimensionless approximations of suitable impedance parameters for the different locomotion tasks of a versatile legged robot is part of future work.

In general optimal or learning gain scheduling controllers such as those presented by Buchli et al. (2011) could be used to design the dynamic behaviour of the robot at each instant in an optimal way. Thus, the four experiments shown here demonstrate how active impedance, by enabling the adjustment of the dynamics of the system on the fly, creates a large potential for new control methods for legged robots.

\section{Discussion}

An important contribution of this work is to discuss how active impedance control can lead to a breakthrough for versatile legged robots. To this end, this section will first provide possible reasons why legged robots are still far from a breakthrough. We will then discuss why springs are currently not ideal to use, and mention the pro and cons of active impedance. Finally, we will propose important future topics of research that will help legged robots become a reality in every-day life.

\subsection{Crucial Limitations of Today's Legged Robots}

As mentioned in the introduction, despite decades of research on legged locomotion, today's robots are still far from being able to move in human environments. Two of the main requirements for such robots are (1) the ability to cope with collisions and non-smooth interactions, since they cannot be avoided in such environments; and (2) the versatility to execute a wide range of tasks to become truly useful assistants. Very few examples of robot designs and their associated control frameworks meet these two requirements. 


\subsection{Springs in Series with the Actuator}

Springs are often added to an actuator to meet the first requirement. However, springs are not an ideal solution to meet the second requirement for the following reasons. A truly versatile robot should be able to execute tasks ranging from a precise and careful manipulation of a delicate object, to locomotion in environments with unperceived obstacles where a soft interaction but also fast reflex motions are required. While some tasks require very precisely controlled joints, others need compliant behaviour. Others require very fast joint motions to react to an external perturbation, e.g. when being pushed or for safety stops. Precise motions at any speed require either a very good model ${ }^{4}$ of the robot and possibly the environment or high gain (i.e. 'stiff') control $^{5}$. In addition, if fast motions are required, as a reaction to an unforeseen event (e.g. side step to keep balance, step reflex or stopping a robot arm in front of a person) a very high actuation bandwidth is required. Compliant behaviour as reaction to an unforeseen perturbation requires low output impedance and is in contradiction to a quick controlled movement without using a model. This fundamentally limits the ability to achieve a quick stop or a sudden reactive movement. Therefore, a compliant robot (or human) needs to have the required bandwidth and high gain control available to be robust in such situations (e.g. a safety stop of a human arm requires immediate stiffening up). For SEA, the spring stiffness fundamentally limits the control bandwidth and this trade-off is fixed at design time. As mentioned in the introduction, VSA might be a possible solution to this problem, however this technology (still) has several limitations. For a VSA, the ability to stop quickly is fundamentally limited by the (usually slow) adaptation of the stiffness. In the case of an active impedance system, the limitations only stem from sensing and actuation delays (actuator physics, data acquisition, data processing), which are, to a large extent, design parameters.

It is important to understand that actuator force is always controlled over a transmission element with finite stiffness. Even stiff actuators without additional springs in series have a certain transmission stiffness that is crucial for force control. In a hydraulic system, for example, this is given by the compressibility of the oil (bulk modulus) and hose elasticity; and in an electric actuator it is the gear box. The transmission stiffness of stiff actuators, however is usually 1-2 orders of magnitude higher than the stiffness of state-of-the-art robots with SEA joints. Figure 10 shows a plot of the linear and rotational stiffness of SEA robots compared with stiff actuator robots. The cylinder stiffness of HyQ is a non-linear function depending on the piston position. Its lowest value is $10^{7} \mathrm{~N} / \mathrm{m}$ (Boaventura (2013), page 115). Mapped into rotational joint space the lowest stiffness becomes $2 \cdot 10^{4} \mathrm{Nm} / \mathrm{rad}$.

To sum up, a versatile robot needs to be able to control its joint stiffness in a wide range. Springs in the structure of a robot including the stiff springs of SEA reduce

\footnotetext{
${ }^{4}$ Note that the fact that models are required for good performance does not address the question where the model comes from. For robots it can sometimes be derived from CAD data, sometimes must be estimated/learned. Humans acquire models by learning.

${ }^{5}$ It is worthwhile discussing these issues in the control theoretic notions of nominal behaviour and disturbance reaction.
} 


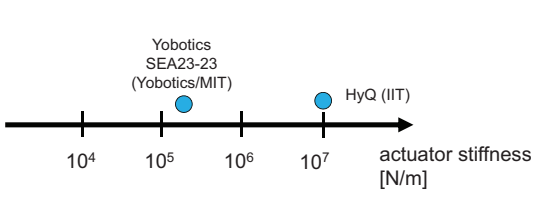

(a) Linear transmission stiffness

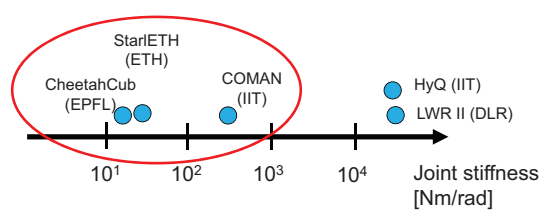

(b) Rotational transmission stiffness

Fig. 10 Transmission stiffness of a selection of robots: (a) Linear transmission stiffness of the Yobotics SEA23-23 actuator (Yobotics) and the hydraulic hip cylinder of HyQ (Semini (2010)). (b) Rotational transmission stiffness of a selection of robots with SEA joints (CheetahCub by Sproewitz et al. (2013), StarlETH by Hutter et al. (2012), COMAN by Tsagarakis et al. (2013)) and stiff actuator robots (KUKA LWR II by Hirzinger et al. (2001) and HyQ by Semini (2010)). It can be seen that the SEA robots have 1-2 orders of magnitude softer joints.

the maximum joint stiffness and control bandwidth; and thus the robot's versatility. We argue that legged robots with active impedance, while certainly not the only solution, are a promising solution that meet both of the above mentioned requirements. Importantly, they are implementable with today's available technology thus putting versatile service robots within immediate reach.

\subsection{Active vs. Passive Compliance}

Active impedance has several advantages when compared to passive springs and dampers. With current advances in actuator, control and computer technology, a wide range of stable stiffness and damping values can be emulated (Boaventura et al. (2013)), which leads to more versatile robots. These values can be adjusted in real-time to swiftly adapt to changing conditions in the environment or task. Furthermore, robots with active impedance can take advantage of any programmable type of impedance (e.g. exponential springs, nonlinear dampers, muscle models, etc.) as described by Boaventura et al. (2012b). A potential drawback of active compliance is low energy efficiency, as no energy can be stored due to a lack of physically compliant elements. Despite this disadvantage we do not consider it as a major problem for the following reasons: One, new methods of high-density energy storage are currently being investigated in various research fields; New compact energy sources will eventually be able to power legged robots for entire days (e.g. Bruce et al. (2012)); Two, new ways of energy recovery such as energy regenerative electronics for electric motors have recently been proposed for joints with active impedance (Seok et al. (2012)). Furthermore, passively compliant elements are only really able to increase energy efficiency of a robot during repetitive motions, such as walking, 
running, scrubbing etc. if the motion frequency is around the resonant frequency of the system ${ }^{6}$.

Two additional important points that should always be discussed when comparing active and passive impedance are: 1) how accurately can an actively-compliant system emulate its passive counterpart; and 2) how well can the active system handle impacts. To address these two points, we have previously performed (and presented in Boaventura et al. (2013)) an experimental comparison between two versions of the HyQ leg, shown in Fig. 11. The actively-compliant version is identical to the one used in HyQ, while the passively-compliant version has a passive spring between the upper and lower leg segment instead of the knee cylinder. Due to the significance of this comparison, we are going to summarize and discuss the experimental results next. For an in-depth discussion of the pro \& cons of active versus passive impedance and details about this comparison we refer the interested reader to Boaventura et al. (2013).

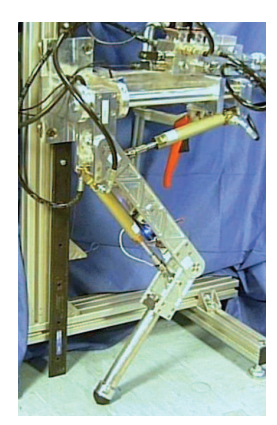

(a) Fully activelycompliant leg

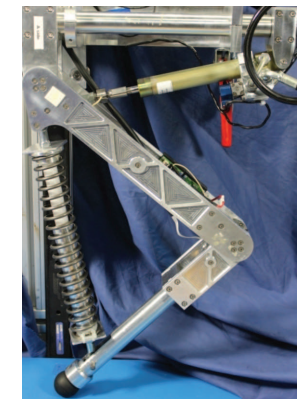

(b) Partially passivelycompliant leg

Fig. 11 HyQ leg fixed to a vertical slider. In (a) the traditional actively-compliant HyQ leg, and in (b) a modified version using a real spring-damper between the hip and the foot. The passivelycompliant version of the leg was only used for comparison and validation purposes. This version of the leg is not used on HyQ.

To best compare the active and passive leg during an impact, both legs were dropped from a height of $25 \mathrm{~cm}$ onto a force plate, where the vertical ground reaction forces $F_{G R}$ were measured. The impedance of the actively-compliant leg was set in software to match the passive version of the leg: stiffness $(K=5250 \mathrm{~N} / \mathrm{m})$, damping $(B=10 N s / m)$, and spring length $(l=0.3 m)$. Also, the weight is roughly the same for both legs. As we can see in Fig. 12, the virtual spring-damper of the actively-compliant leg was able to qualitatively mimic the passive leg behaviour and to handle the impacts. Also, we can notice in the zoom view, at the top right corner, that the impact forces for the actively-compliant leg (dashed red line) are even

\footnotetext{
${ }^{6}$ In recent years, researchers have experimented with clutches and brakes added to SEA to control the release of the stored energy (e.g. Leach et al. (2012); Laffranchi et al. (2014)). However, these prototype actuators have not yet been tested on highly dynamic and versatile legged robots.
} 
smaller than the forces for the leg with the real spring-damper (solid black line). This is an unexpected result, but we have some insights that might explain it. For the real spring-damper assembly, mechanical play and a non-ideal Hookian behaviour for the spring, in addition to a possible increase in the unsprung mass might increase the initial impact forces. On the other hand, for the actively-compliant leg, the internal valve leakage, although not significant, might mitigate the impact force peaks. We are currently preparing experiments that will carefully analyze the behaviour of the actively-compliant leg during an impact and study the influence of components, hydraulic piping, sampling time etc. See also Sect. 6.4 for a further discussion about impact force peaks. In any case, this result is very important since it demonstrates that a purely actively-compliant system can handle impacts as well as (or even better than) passive svstems.

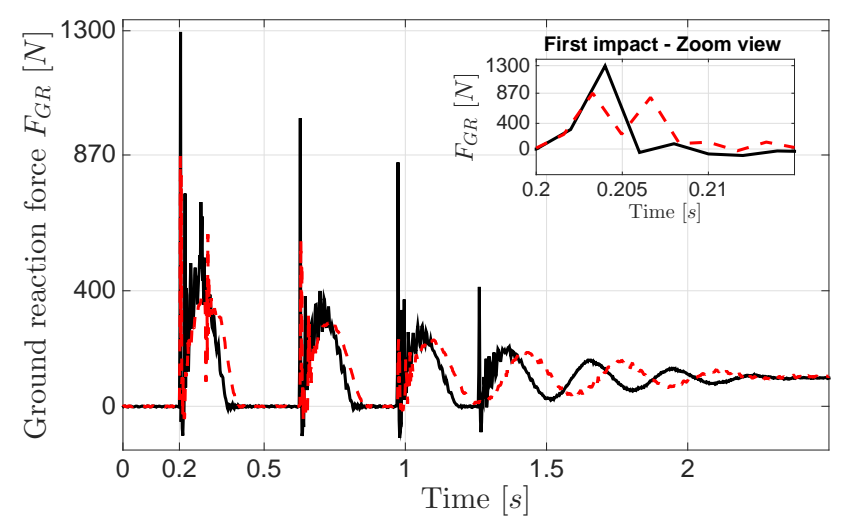

Fig. 12 Vertical ground reaction forces measured with a force plate during a drop test with both fully actively-compliant leg (dashed red line) and the modified passive-spring version of the leg (solid black line). As we can see, the actively-compliant leg can qualitatively emulate the behaviour of its passive counterpart. In addition, in the zoom view of the very first impact, we can notice that the passive version of the leg has impact forces that are even higher than the ones of the active leg.

This experiment also illustrates the main difference between, electric actively compliant systems (such as the aforementioned LWR family for robots) and the herein used actuation system. While from the mechanical design and control point of view the systems are very similar, hydraulics has a threefold advantage over the electric actuation system as currently used in most legged robotics applications ${ }^{7}$. Due to the high output forces at low speeds, hydraulics does not need a high-ratio gear between actuator and load. Thus, (1) the systems can be built with very robust transmission such that even an initial, not fully controlled impact (cf. Fig. 12) is well within the design specifications and no spring is needed for impact protection. (2) The actuation system has a larger actuation bandwidth, which is important for reactive stepping, and this in turn is a key for robust locomotion (Koolen et al. (2012); Barasuol et al. (2013)). (3) It allows for a higher overall control bandwidth at the

\footnotetext{
${ }^{7}$ with a high-ratio reduction gear in series to the motor (see Seok et al. (2013) for an exception.)
} 
forces and torques required for legged robots (i.e. force and position bandwidth measured at the output of the actuator, after gears/transmission). These differences are all very critical in enabling the use of actively compliant actuation for locomotion and other tasks requiring repeated impact loading.

We need to remark that, in general, implementations of active impedance control with an inner torque loop may suffer from some limitations. In particular the fact that the feasible range of stable stiffness and damping values can be strongly limited by the bandwidth of the inner torque loop as well as by the filter and sampling frequency. An extensive analysis on how these aspects influence the stability region of impedance parameters as well as the passivity of the system can be found in Focchi (2013).

\subsection{Stiff Inside and Soft Outside}

This section attempts to give general recommendations about the question where to add compliance into the structure of versatile legged robots and what the parameters of this compliance should be. We introduce the approach stiff inside, soft outside. Stiff inside means that the actuator inside the robot (joints) should be very stiff to maximize active impedance control and position tracking performance. Soft outside means that a certain degree of soft material should be placed at the outside of the robot, i.e. the interface point between the robot and the ground (e.g. at the foot) to reduce force peaks during impact. The required amount of compliance at the outside is related to the performance of the actuation system (e.g. closed-loop bandwidth, sampling delays). The compliance at the interface can absorb the initial force peak during the first few milliseconds of the impact before the controller starts acting. Thus, the faster the actuation, the smaller the required compliance if a maximum tolerated force should not be exceeded (e.g. structural limits of load cells and attachment points). Haddadin et al. (2007) investigated the impact between an actively compliant manipulator arm (DLR lightweight robot III) and humans to analyse the risk of injuries. The paper contains an analysis of the first few milliseconds of the impact and is therefore highly related to this discussion. For manipulators where safety is important, a skin force sensor at the interface can additionally reduce impact peaks. Such a sensor is able to detect a collision before the joint torque sensors, since the measured impact force at the interface is not masked by the inertia of the link. A force sensor at the foot of a legged robot has a similar effect. In hydraulic actuation, the reaction time depends both on the valve bandwidth and the hydraulic stiffness (bulk modulus and oil volumes in cylinder chambers and piping). There is an effective way to limit the maximum (impact) force inside a hydraulic cylinder by adding relief valves that open the chamber to tank above a certain predefined threshold pressure. All the above mentioned points regarding initial impact force peaks, delays, force limiters are part of ongoing studies at IIT. 


\subsection{Discussion Summary}

Now that we understand the limitations introduced by springs we can rethink and adjust future research agendas to focus on the important topics that will lead to a faster introduction of legged robots into everyday life. First of all, torque-controlled robots open up a wide range of control methods besides active impedance, e.g. modelbased control of rigid body dynamics (gravity compensation, inverse dynamics, etc.) and control of contact forces. These are all methods that will lead to improved manipulation and locomotion skills in human environments. One particularly important aspect that helps to support increased performance in both locomotion and manipulation is by reducing the burden on the perception and planning systems. This is achieved by offering a great deal of robustness against imprecise perception or slightly misplanned actions at the high level control level.

Research into optimal selection of stiffness trajectories for a large range of tasks is required. Investigations into how to build more compact and less complex VSAs with fast stiffness adjustment are important because they might be useful in saving energy during repetitive motions. Questions regarding the safety and reliability of active impedance systems were not discussed in detail in this work due to lack of space, but they are important topics that will be investigated in future work. Last but not least, more research into more energy efficient active impedance systems is required.

\section{Conclusions}

We have shown, to the best of our knowledge, for the first time in the academic literature how a legged robot with only active impedance (i.e. without springs) can execute highly dynamic tasks that involve large and impulsive impact forces, such as those generated when running and hopping. Our experiments presented here and elsewhere (Boaventura et al. (2013)) show that it is possible to achieve the same behaviour with a fully actively controlled system as with passive systems. Active impedance, however, also offers the additional advantage of versatility and flexibility, allowing a robot to create the most suitable dynamic behaviour on the fly. The results from this paper show that the assertion that active systems are too slow to control does not hold for the dynamic range that is required for highly dynamic locomotion and interaction tasks on time-, force- and length-scales typical for humans. We consider this approach fundamental to creating a breakthrough in versatile robotic assistants with arms and legs and we have demonstrated that the required control performance is achievable.

\section{Appendix A: Index to Multimedia Extensions}

The multimedia extensions to this article are at: http://www.ijrr.org. 


\begin{tabular}{lll}
\hline Extension & Media Type & Description \\
\hline 1 & Video & The accompanying video material contains scenes of all experiments \\
& & presented in Section 5: (1) flying trot on asphalt, (2) flying trot on \\
& & indoor treadmill, (3) resonant hopping and (4) squat jumps. \\
\hline
\end{tabular}

\section{Acknowledgements}

The authors would like to thank also the other members of IIT's Dynamic Legged Systems Lab who contributed to the success of this project: J. Goldsmith, I. Havoutis, S. Bazeille, H. Khan, B. Rehman, C. Mastalli, M. Camurri and our team of technicians. We also thank G. Medrano-Cerda for his valuable input on control theory, and S. Hubacher and M. Neunert for their support with the experimental setups.

\section{Funding}

This research has been funded by the Fondazione Istituto Italiano di Tecnologia. T. Boaventura is partially funded through the EU FP7-Project BALANCE [grant number 601003]. J. Buchli is supported by a Swiss National Science Foundation professorship.

\section{References}

A. Albu-Schäffer and G. Hirzinger. Cartesian impedance control techniques for torque controlled light-weight robots. In IEEE International Conference on Robotics and Automation (ICRA), pages $657-663,2002$.

A. Albu-Schäffer, C. Ott, and G. Hirzinger. A unified passivity-based control framework for position, torque and impedance control of flexible joint robots. International Journal of Robotics Research (IJRR), 26(1):22-39, 2007.

V. Barasuol, V. J. De Negri, and E. R. De Pieri. WCPG: a central pattern generator for legged robots based on workspaceintentions. In Proceedings of the ASME Dynamic System and Control Conference (DSCC), pages 111-114, 2011.

V. Barasuol, J. Buchli, C. Semini, M. Frigerio, E. R. De Pieri, and D. G. Caldwell. A reactive controller framework for quadrupedal locomotion on challenging terrain. In IEEE International Conference on Robotics and Automation (ICRA), pages 2554-2561., 2013.

S. Bazeille, V. Barasuol, M. Focchi, M. Frigerio, I. Havoutis, D. G. Caldwell, and C. Semini. Quadruped robot trotting over irregular terrain assisted by stereo-vision. Journal of Intelligent Service Robotics, 7(2):67 - 77, 2014.

R. Blickhan. The spring-mass model for running and hopping. Biomechanics, 22:1217-1227, 1989.

T. Boaventura. Hydraulic Compliance Control of the Quadruped Robot HyQ. $\mathrm{PhD}$ thesis, University of Genoa, Italy and Istituto Italiano di Tecnologia (IIT), 2013. 
T. Boaventura, M. Focchi, M. Frigerio, J. Buchli, C. Semini, G. A. Medrano-Cerda, and D. G. Caldwell. On the role of load motion compensation in high-performance force control. In IEEE/RSJ International Conference on Intelligent Robots and Systems (IROS), pages 40664071, 2012a.

T. Boaventura, C. Semini, J. Buchli, M. Frigerio, M. Focchi, and D. G. Caldwell. Dynamic torque control of a hydraulic quadruped robot. In IEEE International Conference on Robotics and Automation (ICRA), pages 1889-1894, 2012b.

T. Boaventura, G. A. Medrano-Cerda, C. Semini, J. Buchli, and D. G. Caldwell. Stability and performance of the compliance controller of the quadruped robot hyq. In IEEE/RSJ International Conference on Intelligent Robots and Systems (IROS), pages 1458-1464, 2013.

Boston Dynamics. Big dog video, 2010. URL http://www.youtube.com/watch?v=cNZPRsrwumQ.

P. G. Bruce, S. A. Freunberger, L. J. Hardwick, and J.-M. Tarascon. Li-O2 and Li-S batteries with high energy storage. Nature Materials, 11:19 - 29, 2012.

J. Buchli, M. Kalakrishnan, M. Mistry, P. Pastor, and S. Schaal. Compliant quadruped locomotion over rough terrain. In IEEE/RSJ International Conference on Intelligent Robots and Systems (IROS), pages 814-820, 2009.

J. Buchli, S. Stulp, E. Theodorou, and S. Schaal. Learning variable impedance control. International Journal of Robotics Research (IJRR), 30(7):820-833, 2011.

M. Buehler, R. Battaglia, R. Cocosco, G. Hawker, J. Sarkis, and K. Yamazaki. SCOUT: a simple quadruped that walks, climbs, and runs. In IEEE International Conference on Robotics and Automation (ICRA), pages 1707-1712, 1998.

E. Burdet, R. Osu, D. Franklin, T. Milner, and M. Kawato. The central nervous system stabilizes unstable dynamics by learning optimal impedance. Nature, 414(6862):446-9, 2001.

G. Cheng, S. Hyon, J. Morimoto, A. Ude, J. G. Hale, G. Colvin, W. Scroggin, and S. C. Jacobsen. CB: A humanoid research platform for exploring neuroscience. Advanced Robotics, 21(10): 1097-1114, 2007.

A. De Luca, A. Albu-Schäffer, S. Haddadin, and G. Hirzinger. Collision detection and safe reaction with the dlr-iii lightweight manipulator arm. In IEEE/RSJ International Conference on Intelligent Robots and Systems (IROS), pages 1623-1630, 2006.

J. Estremera and K. J. Waldron. Thrust control, stabilization and energetics of a quadruped running robot. International Journal of Robotics Research (IJRR), 27(10):1135-1151, 2008.

M. Focchi. Strategies To Improve the Impedance Control Performance of a Quadruped Robot. $\mathrm{PhD}$ thesis, Istituto Italiano di Tecnologia (IIT) and University of Genoa, 2013.

M. Focchi, T. Boaventura, C. Semini, M. Frigerio, J. Buchli, and D. G. Caldwell. Torque-control based compliant actuation of a quadruped robot. In IEEE Int. Workshop on Advanced Motion Control (AMC), 2012.

M. Focchi, V. Barasuol, I. Havoutis, J. Buchli, C. Semini, and D. G. Caldwell. Local reflex generation for obstacle negotiation in quadrupedal locomotion. In Int. Conf. on Climbing and Walking Robots (CLAWAR), 2013.

D. Franklin, E. Burdet, R. Osu, M. Kawato, and T. Milner. Functional significance of stiffness in adaptation of multijoint arm movements to stable and unstable dynamics. Exp Brain Research, $151: 145-157,2003$.

C. Gehring, S. Coros, M. Hutter, M. Bloesch, M. Hoepflinger, and R. Siegwart. Control of dynamic gaits for a quadrupedal robot. In IEEE International Conference on Robotics and Automation (ICRA), pages 3287-3292, 2013.

H. Geyer and H. Herr. A muscle-reflex model that encodes principles of legged mechanics produces human walking dynamics and muscle activities. IEEE Trans Neural Syst Rehabil Eng, 18 (3):263-273, 2010.

S. Haddadin, A. Albu-Schäffer, and G. Hirzinger. Safety evaluation of physical human-robot interaction via crash-testing. In Robotics: Science and System (RSS), pages 217-224, 2007.

I. Havoutis, J. Ortiz, S. Bazeille, V. Barasuol, C. Semini, and D. G. Caldwell. Onboard perceptionbased trotting and crawling with the hydraulic quadruped robot (HyQ). In IEEE/RSJ International Conference on Intelligent Robots and Systems (IROS), pages 6052-6057, 2013. 
A. Herzog, L. Righetti, F. Grimminger, P. Pastor, and S. Schaal. Balancing experiments on a torquecontrolled humanoid with hierarchical inverse dynamics. In IEEE/RSJ International Conference on Intelligent Robots and Systems (IROS), pages 981-988, 2014.

G. Hirzinger, A. Albu-Schäffer, M. Hahnle, I. Schaefer, and N. Sporer. On a new generation of torque controlled light-weight robots. In IEEE International Conference on Robotics and Automation (ICRA), pages 3356-3363, 2001.

N. Hogan. Adaptive control of mechanical impedance by coactivation of antagonist muscles. IEEE Trans Automatic Control, 29:681-690, 1984.

N. Hogan. Impedance control: An approach to manipulation: Part I - Theory. ASME Journal of Dynamic Systems, Measurement, and Control, 107:1-7, 1985a.

N. Hogan. Impedance control: An approach to manipulation: Part II - Implementation. ASME Journal of Dynamic Systems, Measurement, and Control, 107:8-16, 1985 b.

J. W. Hurst. The Role and Implementation of Compliance in Legged Locomotion. PhD thesis, Carnegie Mellon University, 2008.

M. Hutter, C. Gehring, M. Bloesch, M. Hoepflinger, C. D. Remy, and R. Siegwart. Starleth: A compliant quadrupedal robot for fast, efficient, and versatile locomotion. In Int. Conf. on Climbing and Walking Robots (CLAWAR), 2012.

$\mathrm{S}$. Hyon. Compliant terrain adaptation for biped humanoids without measuring ground surface and contact forces. IEEE Transactions on Robotics, 25(1):171-178, 2009.

S. Hyon, T. Yoneda, and D. Suewaka. Lightweight hydraulic leg to explore agile legged locomotion. In IEEE/RSJ International Conference on Intelligent Robots and Systems (IROS), pages 4655-4660, 2013.

IIT, ADVR, DLS lab. Online video of HyQ, March 2014. URL http://youtu.be/ENHvCGrnr2g.

A. J. Ijspeert. Central pattern generators for locomotion control in animals and robots: a review. Neural Networks, 21(4):642-653, 2008.

E. Kandel, J. Schwartz, and T. Jessell. Principles of Neural Science. McGraw-Hill Medical, 4th edition, 2000.

O. Khatib. A unified approach for motion and force control of robot manipulators: The operational space formulation. IEEE Journal of Robotics and Automation, 3(1):43-53, 1987.

J. Kim, Y. Lee, S. Kwon, K. Seo, H. Kwak, H. Lee, and K. Roh. Development of the lower limbs for a humanoid robot. In IEEE/RSJ International Conference on Intelligent Robots and Systems (IROS), pages 4000-4005, 2012.

T. Koolen, T. De Boer, J. Rebula, A. Goswami, and J. Pratt. Capturability-based analysis and control of legged locomotion, part 1: Theory and application to three simple gait models. Int. J. Rob. Res., 31(9):1094-1113, Aug. 2012.

E. Kostamo, M. Focchi, E. Guglielmino, J. Kostamo, C. Semini, J. Buchli, M. Pietola, and D. G. Caldwell. Magnetorheologically damped compliant foot for legged robotic application. Journal of Mechanical Design, 136(2), 2013.

M. Laffranchi, L. Chen, N. Kashiri, J. Lee, N. G. Tsagarakis, and D. G. Caldwell. Development and control of a series elastic actuator equipped with a semi active friction damper for human friendly robots. Robotics and Autonomous Systems, 62(12):1827 - 1836, 2014.

D. Leach, F. Gunther, N. Maheshwari, and F. Iida. Linear multi-modal actuation through discrete coupling. In IEEE/RSJ International Conference on Intelligent Robots and Systems (IROS), pages 2437-2442, 2012.

D. V. Lee, M. R. Isaacs, T. E. Higgins, A. A. Biewener, and C. P. McGowan. Scaling of the spring in the leg during bouncing gaits of mammals. Integr. Comp. Biol., 54(6):1099-1108, 2014.

C. Ott, A. Albu-Schäffer, A. Kugi, S. Stramigioli, and G. Hirzinger. A passivity based cartesian impedance controller for flexible joint robots-part i: Torque feedback and gravity compensation. In IEEE International Conference on Robotics and Automation (ICRA), pages $2659-2665$, 2004.

C. Ott, O. Eiberger, J. Englsberger, M. A. Roa, and A. Albu-Schäffer. Hardware and control concept for an experimental bipedal robot with joint torque sensors. Journal of the Robotics Society of Japan, 30(4):378-382, 2012. 
G. Pratt and M. Williamson. Series elastic actuators. In IEEE/RSJ International Conference on Intelligent Robots and Systems (IROS), pages 399-406, 1995.

J. Pratt, C. Chew, A. Torres, P. Dilworth, and G. Pratt. Virtual model control: An intuitive approach for bipedal locomotion. International Journal of Robotics Research (IJRR), 20(2):129-143, 2001.

M. Raibert. Legged Robots That Balance. The MIT Press, 1986.

M. Raibert, K. Blankespoor, G. Nelson, R. Playter, and the BigDog Team. Bigdog, the roughterrain quadruped robot. In World Congress The International Federation of Automatic Control (IFAC), 2008.

L. Selen, D. Franklin, and D. Wolpert. Impedance control reduces instability that arises from motor noise. J Neurosci, 29(40):12606-16, 2009.

C. Semini. HyQ - Design and Development of a Hydraulically Actuated Quadruped Robot. $\mathrm{PhD}$ thesis, Istituto Italiano di Tecnologia (IIT) and University of Genoa, 2010.

C. Semini, N. G. Tsagarakis, E. Guglielmino, M. Focchi, F. Cannella, and D. G. Caldwell. Design of HyQ - a hydraulically and electrically actuated quadruped robot. Journal of Systems and Control Engineering, 225(6):831-849, 2011.

C. Semini, H. Khan, M. Frigerio, T. Boaventura, M. Focchi, J. Buchli, and D. G. Caldwell. Design and scaling of versatile quadruped robots. In Int. Conf. on Climbing and Walking Robots (CLAWAR), 2012.

C. Semini, V. Barasuol, M. Frigerio, T. Boaventura, and J. Buchli. Is active impedance the key to a breakthrough for legged robots? In International Symposium on Robotics Research (ISRR), 2013.

S. Seok, A. Wang, D. Otten, and S. Kim. Actuator design for high force proprioceptive control in fast legged locomotion. In IEEE/RSJ International Conference on Intelligent Robots and Systems (IROS), pages 1970-1975, 2012.

S. Seok, A. Wang, M. Y. M. Chuah, D. Otten, J. Lang, and S. Kim. Design principles for highly efficient quadrupeds and implementation on the mit cheetah robot. In IEEE International Conference on Robotics and Automation (ICRA), pages 3307-3312, 2013.

R. Shadmer and M. Arbib. A mathematical analysis of the force-stiffness characteristics of muscles in control of a single joint system. Biol. Cybern., 66:463-477, 1992.

A. Sproewitz, A. Tuleu, M. Vespignani, M. Ajallooeian, E. Badri, and A. Ijspeert. Towards Dynamic Trot Gait Locomotion-Design, Control and Experiments with Cheetah-cub, a Compliant Quadruped Robot. International Journal of Robotics Research (IJRR), 32(8):933 - 951, 2013.

K. Sreenath, H.-W. Park, and J. W. Grizzle. Design and experimental implementation of a compliant hybrid zero dynamics controller with active force control for running on MABEL. In IEEE International Conference on Robotics and Automation (ICRA), pages 51-56, 2012.

B. Stephens and C. Atkeson. Modeling and control of periodic humanoid balance using the linear biped model. In IEEE-RAS International Conference on Humanoid Robots (Humanoids), pages 379-384, 2010.

K. Tee, D. Franklin, M. Kawato, T. Milner, and E. Burdet. Concurrent adaptation of force and impedance in the redundant muscle system. Biol. Cybern., 120(1):31-44, 2009.

N. G. Tsagarakis, I. Sardellitti, and D. G. Caldwell. A new variable stiffness actuator (compactvsa): Design and modelling. In IEEE/RSJ International Conference on Intelligent Robots and Systems (IROS), pages 378-383, 2011.

N. G. Tsagarakis, S. Morfey, G. M. Cerda, Z. Li, and D. G. Caldwell. COMpliant huMANoid COMAN: optimal joint stiffness tuning for modal frequency control. In IEEE International Conference on Robotics and Automation (ICRA), pages 673-678, 2013.

B. Vanderborght, A. Albu-Schäffer, A. Bicchi, E. Burdet, D. Caldwell, R. Carloni, M. G. Catalano, O. Eiberger, W. Friedl, G. Ganesh, M. Garabini, M. Grebenstein, G. Grioli, S. Haddadin, H. Hoppner, A. Jafari, M. Laffranchi, D. Lefeber, F. Petit, S. Stramigioli, N. Tsagarakis, M. V. Damme, R. V. Ham, L. C. Visser, and S. Wolf. Variable impedance actuators: a review. Robotics and Autonomous Systems, 61(12):1601-1614, 2013. 
A. Winkler, I. Havoutis, S. Bazeille, J. Ortiz, M. F. Focchi, R. Dillmann, D. G. Caldwell, and C. Semini. Path planning with force-based foothold adaptation and virtual model control for torque controlled quadruped robots. In IEEE International Conference on Robotics and Automation (ICRA), pages 6476-6482, 2014.

Yobotics. Yobotics SEA 23-23 construction instructions. URL http://www.ihmc.us/groups/sea/. 